\title{
A gene-expression-based test can outperform bap1 and p16 analyses in the differential diagnosis of pleural mesothelial proliferations
}

\author{
GRETA ALI $^{1}$, ROSSELLA BRUNO ${ }^{1}$, ANELLO MARCELLO POMA ${ }^{2}$, \\ AGNESE PROIETTI $^{1}$, STEFANO RICCI ${ }^{2}$, ANTONIO CHELLA $^{3}$, FRANCA MELFI $^{4}$, \\ MARCELLO CARLO AMBROGI $^{5}$, MARCO LUCCHI ${ }^{5}$ and GABRIELLA FONTANINI ${ }^{6}$
}

\begin{abstract}
${ }^{1}$ Unit of Pathological Anatomy, Azienda Ospedaliero Universitaria Pisana; ${ }^{2}$ Department of Surgical, Medical, Molecular Pathology and Critical Area, University of Pisa; ${ }^{3}$ Unit of Pneumology; ${ }^{4}$ Unit of Minimally Invasive and Robotic Thoracic Surgery, Robotic Multispeciality Center for Surgery; ${ }^{5}$ Unit of Thoracic Surgery; ${ }^{6}$ Program of Pleuropulmonary Pathology, Azienda Ospedaliero Universitaria Pisana, AOUP, Pisa, I-56126 Tuscany, Italy
\end{abstract}

Received May 14, 2019; Accepted September 25, 2019

DOI: $10.3892 / o l .2019 .11174$

\begin{abstract}
The demonstration of tissue invasion by histology is an essential criterion for the differential diagnosis of benign and malignant mesothelial proliferations. When tissue invasion cannot be identified, the use of ancillary tests is sometimes necessary. Among investigated markers, the loss of BRCA1 associated protein 1 (BAP1) protein expression and the homozygous deletion of p16 have shown $100 \%$ specificity in separating benign and malignant mesothelial lesions. However, beyond the excellent specificity of these two markers, their low sensitivity limits their clinical utility. In this context, a previous study developed and tested a novel tool for use in the differential diagnosis of malignant pleural mesothelioma (MPM) using the NanoString System and a classification algorithm. In the current study, the performance of gene classifiers were compared using BAP1 and p16 testing. p16 FISH and BAP1 immunohistochemistry were performed on the same series of 34 epithelioid MPM and 20 benign pleural lesions, which were previously analyzed by the system. The diagnostic performance of $p 16, \mathrm{BAP} 1$ and our classification models were compared using ROC analysis. It was observed that BAP1 loss and 16 deletion were highly specific for MPM, since they were not detected in benign lesions. However, their AUC values were not completely satisfying (BAP1: 0.8235; p16: 0.7647) particularly due to their low sensitivities. As expected, combining BAP1 and p16 tests increased the
\end{abstract}

Correspondence to: Professor Gabriella Fontanini, Program of Pleuropulmonary Pathology, Azienda Ospedaliero Universitaria Pisana, AOUP, Via Roma 57, Pisa, I-56126 Tuscany, Italy

E-mail: gabriella.fontanini@med.unipi.it

Key words: malignant pleural mesothelioma, mesothelial hyperplasia, $p 16$ fluorescent in situ hybridization, BRCA1 associated protein 1 immunohistochemistry diagnostic sensitivity, thus improving the AUC (0.8824). In the same series of cases, our MPM tool outperformed BAP1 and p16 tests using the 22 and 40 -gene classification models (AUC 22-gene model: 0.9996; AUC 40-gene model: 0.9990). In conclusion, the present gene-expression-based classification exhibited great potential and further validation is required to support these findings in a prospective fashion, in order to provide a solid alternative for pleural proliferation diagnosis.

\section{Introduction}

Malignant pleural mesothelioma (MPM) is a rare and aggressive malignancy arising from the mesothelial cells lining the pleural cavity. There is a clear association between occupational or environmental asbestos exposure, and the development of MPM, with a latency period of about 40 years before disease presentation. Global incidence of MPM has risen steadily over the past decade, and it is predicted to reach the highest peak in $2020(1,2)$. MPM is a heterogeneous tumor, including three main histological subtypes: Epithelioid (60-80\%), sarcomatoid $(<10 \%)$ and mixed $(10-15 \%)(3,4)$.

The definitive MPM diagnosis is mainly based on histopathological examinations of pleural tissues, which could not be sufficiently clear to discriminate MPM neither from secondary tumors involving the pleura nor from benign pleural proliferations (3). Particularly, the differential diagnosis of MPM and benign pleural lesions is a hard task to accomplish, and currently the only criterion to certainly determine the malignancy is the presence of stromal or lung invasion (5). However, it is not always possible to estimate whether stromal invasion is present or not, according to quantitative and qualitative parameters of pleural biopsies and their representativeness of the whole lesion (4). Moreover, for many patients pleural biopsies are not available and diagnosis has to be made on cytological specimens from pleural effusions, whose diagnostic sensitivity is variable ranging from 20 to $70 \%$ (6).

A variety of ancillary tests, mostly based on the evaluation of immunohistochemical markers, have been claimed to 
be useful for separating benign from malignant mesothelial proliferations either on pleural tissues or effusions (7). However, the majority of these markers did not achieve sufficient diagnostic accuracy. Recently, the deletion of the cyclin dependent kinase inhibitor $2 \mathrm{~A}(C D K N 2 A)$ gene, better known as p16, and loss BRCA1 associated protein 1 (BAP1) protein have shown an excellent specificity in separating MPM from pleural mesothelial hyperplasia (MH) (8-12).

p16 is a tumor suppressor gene which is located in chromosome 9p21.3, it regulates cell cycle, and its inactivation results in the enhancement of cell proliferation. Inactivation of pl6 can occur through a homozygous deletion, point mutations or methylation changes. Homozygous deletion of p16, detectable by Fluorescent in Situ Hybridization (FISH), is very common in malignant mesotheliomas, but it has never been described in benign mesothelial proliferations, indicating a specificity of $100 \%$ (8-13). Unfortunately, not all mesotheliomas harbor $p 16$ alterations, and consequently, the sensitivity for epithelioid/biphasic (mixed) and sarcomatoid MPM ranges from approximately 45 to $85 \%$ and 50 to $100 \%$, respectively (11).

BAP1 is a nuclear ubiquitin hydrolase that functions as tumor suppressor; it controls DNA repair, apoptosis promotion, and expression of genes related to cell cycle and cell proliferation. The expression of BAP1 is frequently lost in MPM due to point mutations or chromosomal losses (3p21.1). The lack of immunohistochemical staining is highly specific for MPM, but it is observed only in 60-70 and $15 \%$ of epithelioid/mixed and sarcomatoid mesotheliomas respectively $(8,13)$.

Although the combination of BAP1 and p16 can increase their diagnostic sensitivity, the absence of pl6 deletion or BAP1 loss does not allow to rule out MPM.

In this context, in a previous study (14) our group developed and tested a new tool for MPM differential diagnosis, based on the expression profile of 117 genes that had been reported as deregulated in MPM, including BAPl and pl6. In detail, gene expression levels were determined using the NanoString System (NanoString Technologies) and samples were classified as malignant or benign by the Uncorrelated Shrunken Centroid (USC) classification algorithm. In our precedent study, the USC identified two classification models (22 genes and 40 genes), both able to properly classify all the analyzed pleural samples (14).

The aim of this study was to directly compare the performance of the tool previously identified by our group with BAP1 immunohistochemistry (IHC) and pl6 FISH, in order to evaluate whether it could really improve the differential diagnosis between benign and malignant mesothelial proliferations. In detail, we performed pl6 FISH and BAP1 IHC on the same series of epithelioid MPM and benign pleural lesions, previously analyzed by our system, and assessed the diagnostic performance of each method.

\section{Materials and methods}

Patients. Pleural tissues from 54 patients, comprising 34 epithelioid MPM and 20 pleural MH were analyzed in this study. All patients underwent surgical resection at the Unit of Thoracic Surgery of the University Hospital of Pisa from January 2012 to December 2015. This study was conducted retrospectively conforming to the principles of the Helsinki Declaration of 1975. Clinical information, including patient sex and age, is reported in Table I.

Among the 34 patients with epithelioid MPM, 28 (82.4\%) had a pleurectomy/decortication, whereas the remaining 6 patients (17.6\%) had video-toracoscopic pleural biopsy. Regarding the $20 \mathrm{MH}$ patients, the histological diagnosis of $\mathrm{MH}$ was an incidental finding associated with bollous emphysema and pleural inflammatory effusion.

All tumor samples were formalin-fixed and paraffin embedded (FFPE) for microscopic examination. Histological diagnosis and pathological features were reviewed by two pathologists (GA and GF) according to the WHO 2015 histological and immunohistochemical criteria (15). The most representative paraffin block of each tumor was selected for BAP1 immunohistochemistry and p16 FISH analyses.

Gene expression analysis. Gene expression analysis was performed in our previous study (14) using an nCounter custom codeset including 117 MPM target genes and 6 housekeeping genes, synthesized by NanoString Technologies (NanoString Technologies).

Briefly in the previous work, for each case RNA was purified from four FFPE tissue sections using Qiagen RNeasy FFPE kit (Qiagen) according to manufacturers' instructions. A total of $150 \mathrm{ng}$ RNA was used for NanoString analysis, which was performed in accordance to manufacturers' protocol (NanoString Technologies). Then, for each sample the background noise was calculated on the basis of 8 spike-in negative controls included in the panel. Moreover, the raw NanoString counts of all genes underwent a technical and biological normalization using the nSolver software version 2.5 (NanoString Technologies). The technical normalization, based on 6 spike-in positive controls included in the panel, allows to check on technical variability. On the other hand, the biological normalization, based on the housekeeping genes, allow to correct for differences in RNA input. Only samples which passed both the normalization steps were considered for further statistics and bioinformatics analyses (14).

Immunohistochemistry. IHC was performed on $4 \mu \mathrm{m}$ thick tissue sections that were deparaffinized in xylene and rehydrated using a graded series of ethanol solutions. Sections were then subjected to immunohistochemical staining with a mouse monoclonal primary anti-BAP1 antibody (clone C-4, Santa Cruz Biotechnology; 1: 100 dilution) using the UltraView DAB IHC Detection kit (Ventana Medical System, Inc.). Immunostaining was performed as a fully automated assay using BenchMark ULTRA automated slide stainer (Ventana Medical System, Inc.). Counterstaining was performed with hematoxylin. In all cases, the immunohistochemical evaluation was performed independently by two pathologists (GA and GF) who were blinded to the clinicopathological characteristics of the patients.

Only nuclear expression of BAP1 was considered for evaluation and was scored as positive if there was unambiguous presence of BAP1 expression in mesothelial nuclei without percentage or intensity cutoff values (16-18). The negative controls were carried out by omitting the primary antibody. All the analyzed samples showed internal positive 
Table I. Clinicopathological characteristics of patients.

A, Epithelioid malignant pleural mesothelioma

\begin{tabular}{lc}
\hline Clinicopathological characteristics & n. cases $(\%)$ \\
\hline Age $(\mathrm{N}=34)$ & \\
Range & $40-85$ years \\
Median & 68.5 years \\
Sex $(\mathrm{N}=34)$ & \\
Male & $24(70.6)$ \\
Female & $10(29.4)$ \\
Type of specimen $(\mathrm{N}=34)$ & \\
Pleurectomy/decortication & $28(82.4)$ \\
Pleural biopsy & $6(17.6)$ \\
\hline
\end{tabular}

B, Mesothelial hyperplasia

Clinicopathological characteristics

n. cases $(\%)$

$\begin{array}{lc}\text { Age }(\mathrm{N}=20) & \\ \text { Range } & 18-85 \text { years } \\ \text { Median } & 51.5 \text { years } \\ \text { Sex }(\mathrm{N}=20) & \\ \text { Male } & 15(75.0) \\ \text { Female } & 5(25.0) \\ \text { Type of specimen }(\mathrm{N}=20) & \\ \text { Lung atypical resection } & 9(45.0) \\ \text { Pleural biopsy } & 11(55.0)\end{array}$

controls represented by non-mesothelial BAP1-reactive cells such as fibroblasts, lymphocytes, histiocytes, endothelial cells and pneumocytes. Examples of BAP1 immunostaining are reported in Fig. 1.

pl6 fluorescence in situ hybridization. pl6 deletion was evaluated by FISH using the Vysis LSI CDKN2A(p16) spectrum orange/CEP 9 spectrum green kit (Abbott Molecular) according to the manufacturer's recommendations and as previously described $(19,20)$.

FISH was performed on 4 to $6 \mu \mathrm{m}$ thick paraffin sections of MPM and MH tissues. Before hybridization, paraffin sections were deparaffinized in xylene (3 times, 10 min each), dehydrated by two washing steps of 5 min each in $100 \%$ ethanol and two washing steps of 5 min each in $96 \%$ ethanol, and air-dried at room temperature. Tissue sections were then transferred to a pretreatment solution at $80^{\circ} \mathrm{C}$ for $15 \mathrm{~min}$, followed by a $3 \mathrm{~min}$ wash in purified water, and incubated in a protease solution for $10 \mathrm{~min}$ at $37^{\circ} \mathrm{C}$ to digest proteins. After a brief washing in purified water, the slides were sequentially dehydrated in 70,85 , and $100 \%$ alcohol and air-dried at room temperature. Tissue sections were placed in a Hybrite (Abbott Molecular) for $3 \mathrm{~min}$ at $73^{\circ} \mathrm{C}$ to denature DNA, and probe hybridization was carried out overnight at $37^{\circ} \mathrm{C}$. Tissue sections were washed in $0.1 \% \mathrm{NP} 40 / 2 \mathrm{x} \mathrm{SSC}$ at $76^{\circ} \mathrm{C}$ for $4 \mathrm{~min}$ and then washed in $0.1 \% \mathrm{NP} 40 / 2 \mathrm{x} \mathrm{SSC}$ at room temperature for $1 \mathrm{~min}$. Slides were
Table II. BAP1 IHC and p16 FISH results.

\begin{tabular}{lcc}
\hline Test & $\begin{array}{c}\text { Mesothelial } \\
\text { hyperplasia } \\
\mathrm{N}=20(\%)\end{array}$ & $\begin{array}{c}\text { Malignant pleural } \\
\text { mesothelioma } \\
\mathrm{N}=34(\%)\end{array}$ \\
\hline BAP1 & $20(100)$ & $12(35.3)$ \\
Positive & 0 & $22(64.7)$ \\
Negative & $20(100)$ & $16(47.1)$ \\
$p 16$ & 0 & $18(52.9)$ \\
Negative for deletion & & \\
Positive for deletion & & \\
\hline BAP1, BRCA1 associated protein $1 ;$ IHC, immunohistochemistry; \\
FISH, fluorescent in situ hybridization.
\end{tabular}

mounted with $1.5 \mu \mathrm{g} / \mathrm{ml}$ 4',6-diamidino-2-phenylindole. Tumor samples were scored by 2 independent investigators (GA and AP) who were blinded to the clinicopathological characteristics of the patients and to the immunohistochemical results. Normal cells present in the samples negative for the deletion, such as lymphocytes, fibroblasts, histiocytes, endothelial cells, and pneumocytes, were used as internal positive controls. At least 60 non-overlapping and well-delineated cells were scored for each case. Each specimen was evaluated by the average and the maximum numbers of copies of the $p 16$ gene per cell and the average ratio of the gene to CEP 9 copy numbers. Homozygous deletion was defined by loss of both pl6 gene orange signals when more than $11 \%$ of tumor nuclei showed at least 1 signal CEP 9 green signal (18). Examples of $p 16$ FISH test are reported in Fig. 2.

Statistical analyses. In our previous work, we have identified two classification models (22-gene and 40-gene reported in Table SI), both able to properly classify all the analyzed cases. In this study, the normalized expression levels of the genes included in the two classifiers were selected (14). A partial least square model was used to classify samples with both the 22-gene and 40-gene classifiers by the procedure of the caret $R$ package version 6.0-78. A bootstrap resampling $(n=2000)$ was used to assess the area under the curve (AUC). Also for BAP1, pl6 and their combination, AUC was calculated after bootstrap resampling $(n=2000)$ by the procedure of the pROC $\mathrm{R}$ package version 1.10.0. Positive predictive value (PPV) and negative predictive value (NPV) were assessed for BAP1, p16, the combination of BAP1 and p16 and both gene-classifiers, using the prevalence of our series (0.6296). The association between loss of BAP1 expression and p16 deletion was tested by Fisher's exact test.

\section{Results}

BAPI IHC and pl6 FISH results. BAP1 nuclear expression was observed in all $20 \mathrm{MH}$ cases. Among the 34 MPM cases, 12 showed positive neoplastic cells nuclei, whereas 22 lost BAP1 expression.

As regards $p 16$, all $20 \mathrm{MH}$ cases were negative for $p 16$ deletion. pl6 homozygous deletion was observed in 18 out of 
Table III. Association between BAP1 IHC and p16 FISH.

\begin{tabular}{lcccc}
\hline Mesothelial lesion & $\begin{array}{c}\text { Only } \\
\text { BAP1 loss }(\%)\end{array}$ & $\begin{array}{c}\text { Only } p 16 \\
\text { deletion }(\%)\end{array}$ & $\begin{array}{c}\text { Both BAP1 loss } \\
\text { and } p 16 \text { deletion }(\%)\end{array}$ & $\begin{array}{c}\text { Neither BAP1 loss } \\
\text { nor } p 16 \text { deletion }(\%)\end{array}$ \\
\hline $\begin{array}{l}\text { Mesothelial hyperplasia }(\mathrm{N}=20) \\
\text { Malignant pleural mesothelioma }(\mathrm{N}=34)\end{array}$ & 0 & 0 & 0 & $20(100)$ \\
\hline
\end{tabular}

BAP1, BRCA1 associated protein 1; IHC, immunohistochemistry; FISH, fluorescent in situ hybridization.

Table IV. Performance of BAP1, p16 (alone and in combination) and gene classifiers.

\begin{tabular}{llcrrr}
\hline Test & Sensitivity $(95 \% C I)$ & Specificity $(95 \% C I)$ & AUC (95\% CI) & PPV & NPV \\
\hline BAP1 & $0.6471(0.4706-0.7941)$ & 1 & $0.8235(0.7353-0.8971)$ & 1 & 0.6250 \\
p16 & $0.5294(0.3529-0.7059)$ & 1 & $0.7647(0.6765-0.8529)$ & 1 & 0.5556 \\
BAP1 and $p 16$ & $0.7647(0.6176-0.8824)$ & 1 & $0.8824(0.8088-0.9412)$ & 1 & 0.7144 \\
22 genes & $0.9784(0.9018-1)$ & $0.9987(0.9682-1)$ & $0.9996(0.9945-1)$ & 0.9992 & 0.9645 \\
40 genes & $0.9701(0.8817-1)$ & $0.9957(0.9338-1)$ & $0.9990(0.9894-1)$ & 0.9974 & 0.9515 \\
\hline
\end{tabular}

BAP1, BRCA1 associated protein 1; AUC, area under the curve; PPV, positive predictive value; NPV, negative predictive value; CI, confidence interval.
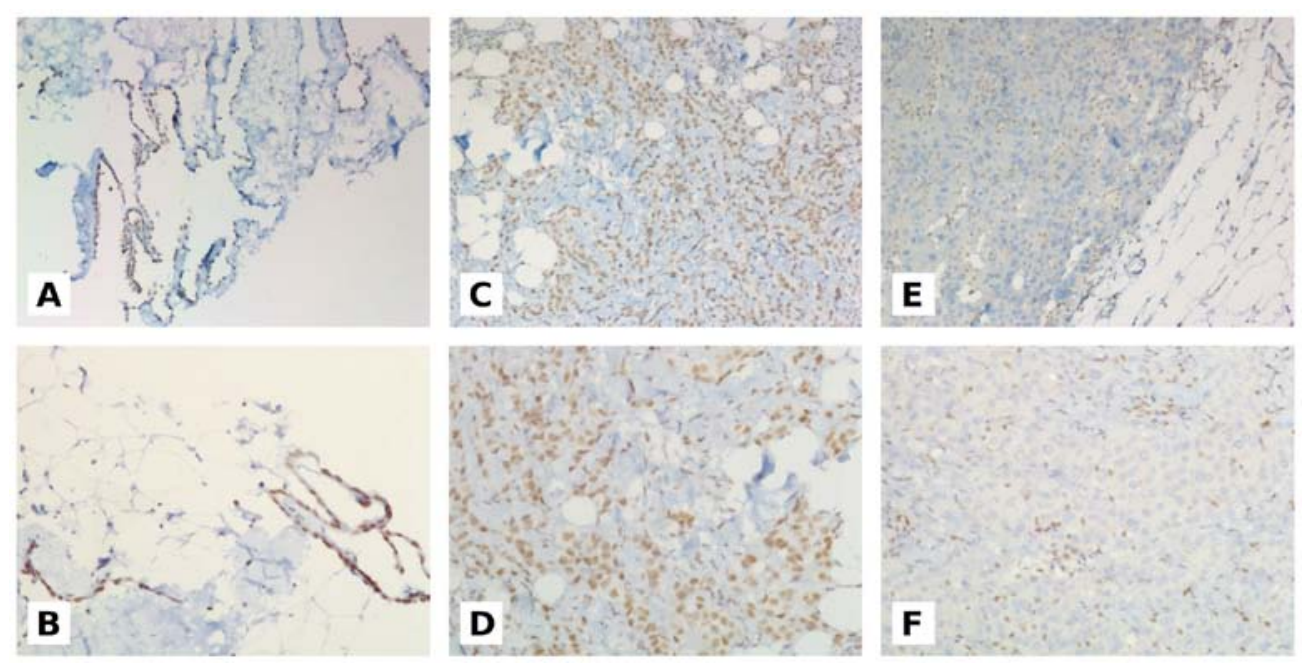

Figure 1. BAP1 immunohistochemistry. (A and B) presence of BAP1 immunostaining in reactive pleural cells and in inflammatory cells in a case of MH (A) original magnification x10; (B) original magnification x20); (C and D) retained BAP1 expression in a MPM case (C) original magnification x10; (D) original magnification x20); (E and F) absence of BAP1 nuclear staining in MPM cells and presence of nuclear staining in adjacent benign cells (internal control) (E) original magnification x10; (F) original magnification x20). BAP1, BRCA1 associated protein 1; MH, mesothelial hyperplasia; MPM, malignant pleural mesothelioma.
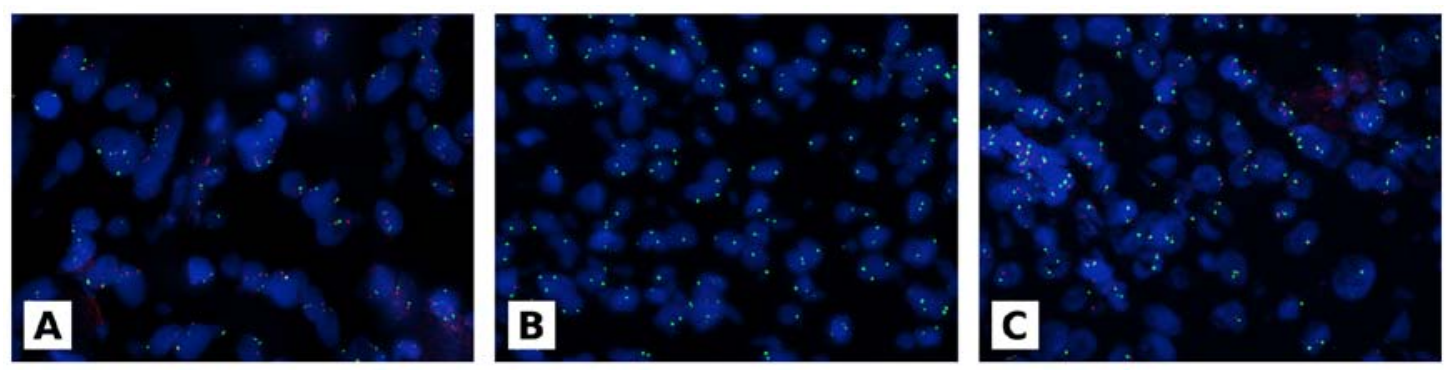

Figure 2.p16 FISH images. (A) CDK2NA (p16) deletion-negative pattern in mesothelial cells in a case of $\mathrm{MH}$; (B) homozygous deletion pattern for $C D K N 2 A$ ( $p 16$ ) in MPM cells; (C) example of MPM case showing CDK2NA (p16) deletion-negative pattern (original magnification x60). CDK2NA, cyclin dependent kinase inhibitor 2A; MH, mesothelial hyperplasia; MPM, malignant pleural mesothelioma. 
34 MPM cases, whereas 16 out of 34 were negative for the deletion. Furthermore, there was no association between BAP1 loss and p16 deletion. Details are reported in Tables II and III.

Comparison among BAPI IHC, p16 FISH, and gene expression panel. The AUC for BAP1 and p16 was 0.8235 and 0.7647 respectively. Although the combination of BAP1 and p16 produced a higher AUC than those obtained with a single biomarker (0.8824), both gene-classifiers reached better AUC, 0.9996 and 0.9990 for the 22-gene and 40-gene classifier respectively. Sensitivity, specificity, PPV and NPV of BAP1, p16 and gene-classifiers in discriminating MH and MPM are summarized in Table IV.

\section{Discussion}

Differential diagnosis between epithelioid MPM and reactive $\mathrm{MH}$ is one of the most challenging diagnostic issues. To date, the best criterion to ascertain the malignancy of pleural lesions is the presence of stromal or lung invasion, which is not always easy to evaluate $(3,15,21)$. So the analyses of $p 16$ gene deletion and BAP1 loss of expression are recommended (8-13). Overall, BAP1 and p16 examinations do not allow the detection of all MPM cases, even combining the two assays, since they are altered only in a proportion of mesotheliomas (8). In a previous study we identified two classification models based on the expression profile of 22 and 40 genes specifically deregulated in MPM, which perfectly worked in discriminating epithelioid MPM from benign lesions (14).

In the present study, we compared the performance of our gene classifiers with BAP1 and p16 testing. We observed that both BAP1 loss and p16 deletion were highly specific for MPM, since they were never detected in benign lesions. However, their AUC values were not completely satisfying (BAP1: 0.8235 ; $p 16$ : 0.7647 ) particularly due to their low sensitivities, in fact 8 MPM cases $(23.5 \%)$ were negative for $p 16$ deletion as well as positive for BAP1 expression. As expected, combining BAP1 and p16 tests increased the diagnostic sensitivity, thus improving the AUC (0.8824). All these results were in agreement with other previously published studies $(12,13,16,22,23)$. Furthermore, we confirmed that there was no association between BAP1 loss and p16 deletion $(13,18,24)$.

In our series, both the 22- and 40-gene expression classifiers outperformed BAP1 and p16 tests (AUC 22-gene model: 0.9996; AUC 40-gene model: 0.9990).

BAP1 and p16 are undoubtedly valuable MPM biomarkers, but, as confirmed in this study, a multi-marker approach seemed to better overcome the great heterogeneity of this tumor (7).

Our MPM tool requires a low input of starting material comparable or even less than the one necessary for BAP1 and p16 evaluation. Moreover, our system allows to obtain a faster analysis and an easier interpretation of results. In fact, IHC and FISH tests require a tissue section for each marker and can be influenced by several pre-analytical factors. Moreover, the interpretation of FISH test can be quite challenging because it requires highly skilled staff.

On the other hand, the MPM tool is highly reproducible, and almost completely automatized $(14,25)$. It could also be even more informative, due to the inclusion of genes with a crucial role in cancer development, and progression (14); some of which also correlate with MPM prognosis and are potential therapeutic targets $(26,27)$.

Our gene expression classifiers proved a great potential as a diagnostic tool. The encouraging results on histological specimens suggest that a prospective validation is warranted to concretely evaluate the use of the 117 gene panel in the clinical context.

\section{Acknowledgements}

Not applicable.

\section{Funding}

No funding was received.

\section{Availability of data and materials}

The datasets used and/or analyzed during the present study are available from the corresponding author on reasonable request.

\section{Authors' contributions}

GA, GF and RB contributed to study design, data collection and interpretation. AMP contributed to statistical and data analysis. GA, GF, AP and SR contributed to histological revision of cases, immunohistochemistry and FISH analysis. AC, FM, MCA, ML defined the study population, designed the study, drafted and critical revised the manuscript. All authors discussed the results and contributed to the final manuscript.

\section{Ethics approval and consent to participate}

This study was retrospectively conducted in accordance to the principles of the Helsinki Declaration of 1975 and was approved by Comitato Etico di Area Vasta Nord-Ovest per la Sperimentazione Clinica. Only archival and anonymous samples were included, no protected health information was used and informed consents were obtained from patients.

\section{Patient consent for publication}

Not applicable.

\section{Competing interests}

The authors declare that they have no competing interests.

\section{References}

1. Scherpereel A, Astoul P, Baas P, Berghmans T, Clayson H, de Vuyst P, Dienemann H, Galateau-Salle F, Hennequin C, Hillerdal G, et al: Guidelines of the European respiratory society and the European society of thoracic surgeons for the management of malignant pleural mesothelioma. Eur Respir J 35: 479-495, 2010.

2. Sekido Y: Molecular pathogenesis of malignant mesothelioma. Carcinogenesis 34: 1413-1419, 2013.

3. Husain AN, Colby TV, Ordóñez NG, Allen TC, Attanoos RL, Beasley MB, Butnor KJ, Chirieac LR, Churg AM, Dacic S, et al: Guidelines for pathologic diagnosis of malignant mesothelioma 2017 update of the consensus statement from the international mesothelioma interest group. Arch Pathol Lab Med 142: 89-108, 2018. 
4. Alì G, Bruno R and Fontanini G: The pathological and molecular diagnosis of malignant pleural mesothelioma: A literature review. J Thorac Dis 10 (Suppl 2): S276-S284, 2018.

5. Galateau-Salle F, Churg A, Roggli V and Travis WD; World Health Organization Committee for Tumors of the Pleura: The 2015 World Health Organization Classification of Tumors of the Pleura: Advances since the 2004 classification. J Thorac Oncol 11: 142-154, 2016.

6. Hjerpe A, Ascoli V, Bedrossian C, Boon M, Creaney J, Davidson B, Dejmek A, Dobra K, Fassina A, Field A, et al: Guidelines for cytopathologic diagnosis of epithelioid and mixed type malignant mesothelioma. Complementary statement from the International Mesothelioma Interest Group, also endorsed by the International Academy of Cytology and the Papanicolaou Society of Cytopathology. Cytojournal 12: 26, 2015.

7. Bruno R, Alì G and Fontanini G: Molecular markers and new diagnostic methods to differentiate malignant from benign mesothelial pleural proliferations: A literature review. J Thorac Dis 10 (Suppl 2): S342-S352, 2018

8. Churg A, Sheffield BS and Galateau-Salle F: New markers for separating benign from malignant mesothelial proliferations: Are we there yet? Arch Pathol Lab Med 140: 318-321, 2016.

9. Ladanyi M: Implications of P16/CDKN2A deletion in pleural mesotheliomas. Lung Cancer 49 (Suppl 1): S95-S98, 2005.

10. Illei PB, Ladanyi M, Rusch VW and Zakowski MF: The use of CDKN2A deletion as a diagnostic marker for malignant mesothelioma in body cavity effusions. Cancer 99: 51-56, 2003.

11. Dacic S, Kothmaier H, Land S, Shuai Y, Halbwedl I, Morbini P, Murer B, Comin C, Galateau-Salle F, Demirag F, et al: Prognostic significance of $\mathrm{p} 16 / \mathrm{cdkn} 2 \mathrm{a}$ loss in pleural malignant mesotheliomas. Virchows Arch 453: 627-635, 2008.

12. Chung CT, Santos GD, Hwang DM, Ludkovski O, Pintilie M, Squire JA and Tsao MS: FISH assay development for the detection of p16/CDKN2A deletion in malignant pleural mesothelioma. J Clin Pathol 63: 630-634, 2010.

13. Sheffield BS, Hwang HC, Lee AF, Thompson K, Rodriguez S, Tse CH, Gown AM and Churg A: BAP1 immunohistochemistry and p16 FISH to separate benign from malignant mesothelial proliferations. Am J Surg Pathol 39: 977-982, 2015.

14. Bruno R, Alì G, Giannini R, Proietti A, Lucchi M, Chella A, Melfi F, Mussi A and Fontanini G: Malignant pleural mesothelioma and mesothelial hyperplasia: A new molecular tool for the differential diagnosis. Oncotarget 8: 2758-2770, 2017.

15. Travis WD, Brambilla E, Burke AP, Marx A and Nicholson AG Introduction to the 2015 World Health Organization Classification of tumors of the lung, pleura, thymus, and heart. J Thorac Oncol 10: 1240-1242, 2015.

16. Cigognetti M, Lonardi S, Fisogni S, Balzarini P, Pellegrini V, Tironi A, Bercich L, Bugatti M, Rossi G, Murer B, et al: BAP1 (BRCA1-associated protein 1) is a highly specific marker for differentiating mesothelioma from reactive mesothelial proliferations. Mod Pathol 28: 1043-1057, 2015.
17. McGregor SM, Dunning R, Hyjek E, Vigneswaran W, Husain AN and Krausz T: BAP1 facilitates diagnostic objectivity, classification, and prognostication in malignant pleural mesothelioma. Hum Pathol 46: 1670-1678, 2015.

18. McGregor SM, McElherne J, Minor A, Keller-Ramey J, Dunning R, Husain AN, Vigneswaran W, Fitzpatrick C and Krausz T: BAP1 immunohistochemistry has limited prognostic utility as a complement of CDKN2A (p16) fluorescence in situ hybridization in malignant pleural mesothelioma. Hum Pathol 60: 86-94, 2017.

19. Chiosea S, Krasinskas A, Cagle PT, Mitchell KA, Zander DS and Dacic S: Diagnostic importance of 9p21 homozygous deletion in malignant mesotheliomas. Mod Pathol 21: 742-747, 2008.

20. Monaco SE, Shuai Y, Bansal M, Krasinskas AM and Dacic S: The diagnostic utility of p16 FISH and GLUT-1 immunohistochemical analysis in mesothelial proliferations. Am J Clin Pathol 135: 619-627, 2011.

21. Churg A, Colby TV, Cagle P, Corson J, Gibbs AR, Gilks B, Grimes M, Hammar S, Roggli V and Travis WD: The separation of benign and malignant mesothelial proliferations. Am J Surg Pathol 24: 1183-1200, 2000.

22. Husain AN: Mesothelial proliferations: Useful marker is not the same as a diagnostic one. Am J Clin Pathol 141: 152-153, 2014.

23. Nasu M, Emi M, Pastorino S, Tanji M, Powers A, Luk H, Baumann F, Zhang YA, Gazdar A, Kanodia S, et al: High Incidence of Somatic BAP1 alterations in sporadic malignant mesothelioma. J Thorac Oncol 10: 565-576, 2015.

24. Hwang HC, Sheffield BS, Rodriguez S, Thompson K, Tse CH, Gown AM and Churg A: Utility of BAP1 immunohistochemistry and p16 (CDKN2A) FISH in the diagnosis of malignant mesothelioma in effusion cytology specimens. Am J Surg Pathol 40: 120-126, 2016

25. Tsang HF, Xue VW, Koh SP, Chiu YM, Ng LP and Wong SC: NanoString, a novel digital color-coded barcode technology: Current and future applications in molecular diagnostics. Expert Rev Mol Diagn 17: 95-103, 2017.

26. Linton A, Cheng YY, Griggs K, Schedlich L, Kirschner MB, Gattani S, Srikaran S, Chuan-Hao Kao S, McCaughan BC, Klebe S, et al: An RNAi-based screen reveals PLK1, CDK1 and NDC80 as potential therapeutic targets in malignant pleural mesothelioma. Br J Cancer 110: 510-519, 2014.

27. Kato T, Lee D, Wu L, Patel P, Young AJ, Wada H, Hu HP, Ujiie H, Kaji M, Kano S, et al: SORORIN and PLK1 as potential therapeutic targets in malignant pleural mesothelioma. Int J Oncol 49: 2411-2420, 2016. 